\title{
Reconciling the electronic and geometric corrugations of the hexagonal boron nitride and graphene nanomeshes
}

\author{
William C. McKee, ${ }^{1}$ Vincent Meunier, ${ }^{2}$ and $Y e X u^{1 *}$ \\ ${ }^{1}$ Department of Chemical Engineering, Louisiana State University, Baton Rouge, Louisiana 70803, USA \\ ${ }^{2}$ Department of Physics, Applied Physics, and Astronomy, Rensselaer Polytechnic Institute, Troy, New York 12180, USA
}

\begin{abstract}
Monolayer hexagonal boron nitride on $\mathrm{Rh}(111)$ and graphene on $\mathrm{Ru}(0001)$ illustrate a trend of divergence between the density functional theory (DFT) calculated geometric corrugation, and scanning tunneling microscope (STM) measured apparent corrugation, of metal-supported 2D films that feature chemically distinct regions. Notably, the geometric and apparent corrugations differ by up to $2 \AA$ for boron nitride/Rh(111), whereas both the DFT-simulated and the experimentally observed STM images agree in the apparent corrugation over a wide range of bias voltages. The disparity is due to unequal contributions of the low/high-lying atoms to the local density of states in the vicinity of the Fermi level. This phenomenon has important implications for the structural characterization of certain supported 2D films, which are being explored for novel electronic and material applications.
\end{abstract}

\section{Keywords}

boron nitride; graphene; nanomesh; density functional calculations; STM simulation

*yexu@1su.edu 
Moiré-structured monolayer graphene (g) and hexagonal boron nitride (h-BN) films supported on transition metals have received considerable attention recently [1-3] due to their fascinating electronic properties $[4,5]$, and their potential to act as substrates that promote the self-assembly of molecules and metal clusters relevant to material and catalytic applications [6-8]. However, several details regarding the physical characteristics of these nanomesh structures, such as their periodicity and corrugation, remain unsettled. Here we take h-BN/Rh(111) and $g / \operatorname{Ru}(0001)$ as examples, and perform self-consistent van der Waals (vdW) corrected density functional theory (DFT) calculations for both structural optimization and scanning tunneling microscopy (STM) image simulation to demonstrate a trend of divergence between the STM-measured apparent corrugation vs. actual geometric corrugation of nanomeshes formed by 2D films on transition metal surfaces. In particular, we show that relatively small apparent corrugations observed for the 2D nanomeshes in STM experiments are fully compatible with much larger geometric corrugations as predicted by DFT. This is due to a systematic electronic effect inherent in these corrugated 2D films. STM is now a mainstay surface characterization technique and is used, often implicitly, to evaluate the choice of density functional and the validity of structural predictions in theoretical modeling. Therefore this trend has important implications for the understanding and prediction of the surface structure-function relationship of corrugated 2D films, such as the ability of the h-BN and graphene nanomeshes to trap macromolecules $[2,9]$ and metal clusters $[6,7]$, where the effect of the surface corrugation on global diffusion is important [10-14].

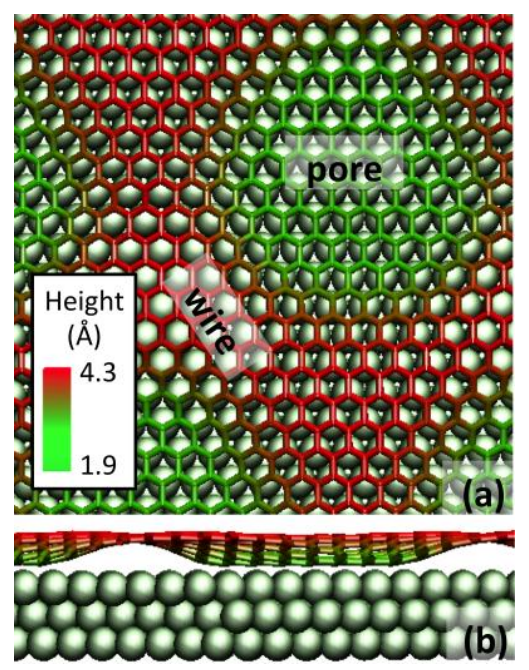

FIG. 1. (a) Top and (b) side views of the DFT-optimized structure (using the optB86b vdW functional) of the h-BN nanomesh on $\mathrm{Rh}(111)$. The nanomesh is colored according to its height above $\mathrm{Rh}(111)$ (pore region in green/lighter shades and wire region in red/darker shades). Rh atoms are shown as spheres. 
Recently Patterson et al. identified a discrepancy in the experimental and theoretical values of the corrugation of the h-BN/Rh(111) nanomesh [15], which consists of alternating low-lying "pore" and high-lying "wire" regions [2] (Fig. 1). The apparent corrugation in STM at a sample voltage of $+1.0 \mathrm{~V}$ was found to be $0.5 \AA$, consistent with Brihuega et al. who reported the apparent corrugation of $\mathrm{h}-\mathrm{BN} / \mathrm{Rh}(111)$ to be $0.5 \sim 1.0 \AA$ over a sample voltage range of -5 to $+1 \mathrm{~V}$ [7]. However, the geometric corrugation calculated using DFT with several different exchangecorrelation functionals ranges from 2.2 to $2.6 \AA$ [15]. Furthermore, because the h-BN/Rh(111) nanomesh is much more effective than, e.g. $g / \operatorname{Ru}(0001)$, at nucleating $\mathrm{Au}$ nanoclusters and preventing them from agglomerating [15, 16], a corrugation that is comparable to $g / R u(0001)(0.15 \sim 1.5 \AA$ based on different experimental techniques [17-19]) seems puzzling.

Here we examine the disagreement between the STM and DFT estimates of the $\mathrm{h}-\mathrm{BN} / \mathrm{Rh}(111)$ nanomesh corrugation in detail, and establish that an apparent corrugation of $0.5 \AA$ is fully consistent with an actual geometric corrugation of $>2.0 \AA$. In particular, we show that the chemically distinct low/high-lying regions of the h-BN nanomesh make unequal contributions to the local density of states (LDOS) near the Fermi energy $\left(\mathrm{E}_{\mathrm{F}}\right)$, similar to what has been previously noted for the $\mathrm{g} / \mathrm{Ru}(0001)$ nanomesh [20]. This leads to an apparent electronic corrugation that differs significantly from the geometric corrugation in both cases, a factor that should be considered in addition to voltage dependencies and tip effects [21] when interpreting STM measurements.

The h-BN/Rh(111) and $g / R u(0001)$ nanomeshes are optimized using self-consistent periodic DFT calculations based on the optB86b vdW functional of Klimeš et al. [22], and the projected augmented wave method as implemented in the VASP code (version 5.3) [23, 24]. The Kohn-Sham eigenstates are expanded in a plane-wave basis up to an energy cutoff of $400 \mathrm{eV}$. The Methfessel-Paxton scheme is used to smear the electronic states with a

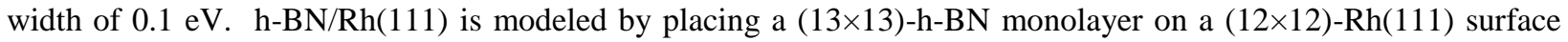
(i.e., 13-on-12) [15]. For $\mathrm{g} / \mathrm{Ru}(0001)$, the unit cell is comprised of a $(12 \times 12)$ graphene monolayer on an $(11 \times 11)$ $\mathrm{Ru}(0001)$ surface [25]. 25-on-23 and 11-on-10 $\mathrm{g} / \mathrm{Ru}(0001)$ structures have also been proposed, but their DFTcalculated corrugations are similar [26, 27]. The optimization of $h-B N / R h(111)$ and $g / R u(0001)$ both proceed from an initially flat film on one side of a three-layer thick metal slab. In each instance the unit cell is based on the equilibrium lattice constant of the transition metal resulting in an initially strained $2 \mathrm{D}$ film, and the strain is partially relieved upon optimization to the corrugated minimum-energy structure. The optB86b lattice constants for $\mathrm{Rh}$ and 
$\mathrm{Ru}(\mathrm{a}=3.803 \AA$, and $\mathrm{a} / \mathrm{c}=2.702 / 4.266 \AA)$ closely match experiment $(\mathrm{a}=3.80 \AA$, and $\mathrm{a} / \mathrm{c}=2.70 / 4.266 \AA)[28]$. The atoms in the nanomesh and the topmost metal layer are relaxed until the force in each relaxed degree of freedom fall below $0.03 \mathrm{eV} / \AA$. At least $10 \AA$ of vacuum space is included between the top of the optimized nanomeshes and the neighboring metal slabs in the $\mathrm{z}$ direction, with electrostatic decoupling applied in the $\mathrm{z}$ direction [29]. Due to the large size of the unit cells, reciprocal space is sampled at the $\Gamma$ point only during geometry optimization. The electronic structures of the optimized cells are subsequently evaluated on a $3 \times 3 \times 1$ Monkhorst-Pack k-point grid while keeping the structures frozen.

For the optimized $\mathrm{h}-\mathrm{BN} / \mathrm{Rh}(111)$ nanomesh, the minimum pore-Rh distance is found to be $2.14 \AA$. The highest nanomesh atom is an $\mathrm{N}$ in the wire region, and the lowest a B atom in the pore region. Their height difference (the maximum corrugation), $2.39 \AA$ based on optB86b, is in fair agreement with the corresponding values based on LDA $(2.65 \AA)$ and GGA-PBE $(2.20 \AA$ ) [15], but differs significantly from the $0.5 \AA$ and $1.2 \AA$ obtained by Laskowski et al. [30] and Diaz et al. [31] using the WC and revPBE+D3 functionals, respectively. Recently Iannuzzi el al. have also reported different geometric corrugations for h-BN/Rh(111) based on the vdW-DF of Dion et al., revPBE+D3, and PBE-rVV10 (0.9, 1.1, and $2.3 \AA$, respectively), and concluded that PBE-rVV10, another self-consistent vdW functional, is best suited to describing the structure of the h-BN nanomesh and the adsorption properties of phthalocyanines thereon [9]. The large spread in the h-BN corrugation values given by the different functionals highlights the need for careful validation of the theoretical results by comparison with experiment. We therefore also simulated STM images for the $\mathrm{h}-\mathrm{BN} / \mathrm{Rh}(111)$ nanomesh over a range of bias voltage for comparison with the available experimental data.

STM images are simulated via the Tersoff-Hamann [32] formalism at an isodensity contour of $2.0 \times 10^{-3}$ At this contour value, the simulated apparent corrugation for $\mathrm{h}-\mathrm{BN} / \mathrm{Rh}(111)$ is $0.6 \AA$ at a sample voltage of $+1.0 \mathrm{~V}$ and $1.1 \AA$ at $-1.0 \mathrm{~V}$, in close agreement with the experimental values of $0.5 \AA$ at $+1.0 \mathrm{~V}[15]$ and $0.88 \AA$ at $-1.0 \mathrm{~V}$ [9]. Furthermore, both the predicted apparent corrugation and its voltage dependence agree well with the experimental results of Brihuega et al. [7] in the range of $-1.0 \sim+2.0 \mathrm{~V}$. Thus, the significant geometric corrugation in $\mathrm{h}-\mathrm{BN} / \mathrm{Rh}(111)$ predicted by DFT is compatible with the shallow corrugations seen in STM. In STM measurements the precise geometry (and hence electronic structure) of the tip is generally unknown, and may differ from tip to tip. The Tersoff-Hamann formalism, unlike more sophisticated approaches (e.g. the Bardeen method), does not account for effects due to the STM tip, but has the interpretive advantage that the resulting STM images 
reflect the electronic structure of the surface alone. The inclusion of the tip in STM simulation would likely further reduce the apparent surface corrugation and is beyond the scope of the present work.

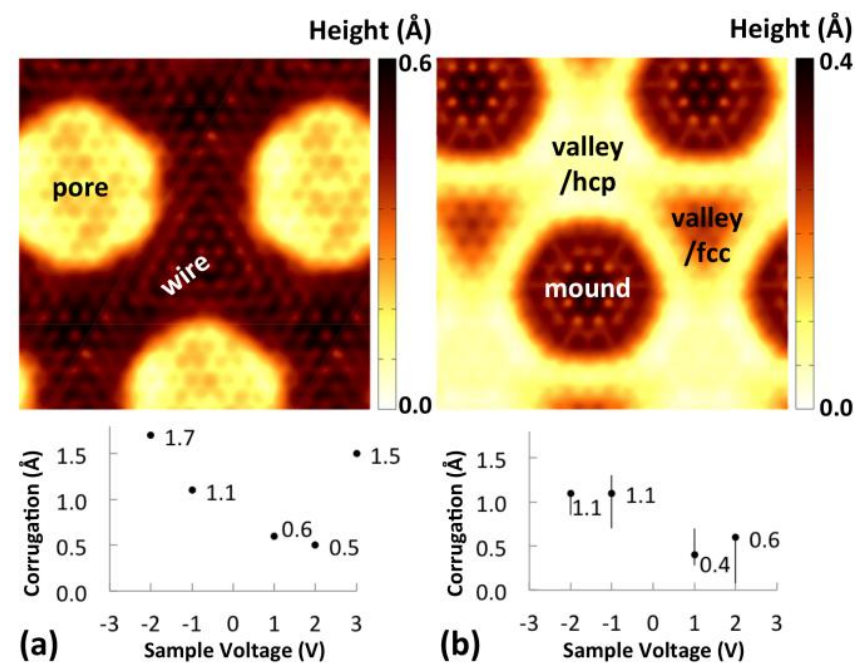

FIG. 2. Simulated STM images at a sample voltage of $+1.0 \mathrm{~V}$ (top panel), and maximum apparent corrugations at various sample voltages (bottom panel, measured between highest atom in wire/mound region and lowest atom in pore/valley region), for (a) h$\mathrm{BN} / \mathrm{Rh}(111)$ and (b) $\mathrm{g} / \mathrm{Ru}(0001)$. Different regions of the two nanomeshes are labeled. The height scale is relative to the lowest tip position in each simulation. The $+3 \mathrm{~V}$ point for $\mathrm{h}-\mathrm{BN} / \mathrm{Rh}(111)$ is due to inverted contrast (see text). Braces around the apparent corrugations of $\mathrm{g} / \mathrm{Ru}(0001)$ reflect the range of experimentally determined values from Ref. [26].

Following Tersoff-Hamann [32], the tunneling current is proportional to the LDOS at the position of the tip. Hence, in the constant current mode, the tip is envisioned as moving along an isodensity contour derived from a partial charge density arising from the states in a given energy range on one side of $\mathrm{E}_{\mathrm{F}}$ chosen to mimic the bias voltage (e.g., $+1.0 \mathrm{~V}$ sample voltage corresponds to states in the $\mathrm{E}_{\mathrm{F}} \sim \mathrm{E}_{\mathrm{F}}+1.0 \mathrm{eV}$ range). In the $\pm 2.0 \mathrm{eV}$ range about the $E_{F}$, the $h-B N$ nanomesh contribution to the LDOS originates mainly from its $p_{z}$ states, with the $\mathrm{N}_{\mathrm{z}}$ projected DOS (PDOS) far outweighing the B $\mathrm{p}_{\mathrm{z}}$ PDOS. The $\mathrm{p}_{\mathrm{x}}$ and $\mathrm{p}_{\mathrm{y}}$ states of the $\mathrm{B}$ and $\mathrm{N}$ atoms lie too low in energy (starting at about $-5 \mathrm{eV}$ relative to $\mathrm{E}_{\mathrm{F}}$ ) to be pertinent to the current discussion of STM imaging. Importantly, the respective contributions of the low/high-lying atoms to the LDOS are not equal. The $\mathrm{p}_{\mathrm{z}}$ states of the low-lying atoms dominate in the vicinity of $\mathrm{E}_{\mathrm{F}}\left(\mathrm{Fig}\right.$. 3(a)), indicating that the $\mathrm{BN}$ states in the vicinity of $\mathrm{E}_{\mathrm{F}}$ are localized in the pore regions of the nanomesh. Hence the electronic corrugation arising from the LDOS near $\mathrm{E}_{\mathrm{F}}$ differs significantly from the geometric corrugation, which explains the discrepancy between the STM-measured apparent corrugation and DFT-calculated geometric corrugations for h-BN/Rh(111). We emphasize that this "opposing electronic corrugation" exists only in the LDOS. The electronic corrugation of the nanomesh arising from the total charge density (i.e., arising from all occupied states) closely mimics the actual geometric corrugation. Therefore the 
inability of STM measurements to determine the geometric corrugation of $\mathrm{h}-\mathrm{BN} / \mathrm{Rh}(111)$ arises from its limited sampling of states near the $\mathrm{E}_{\mathrm{F}}$.

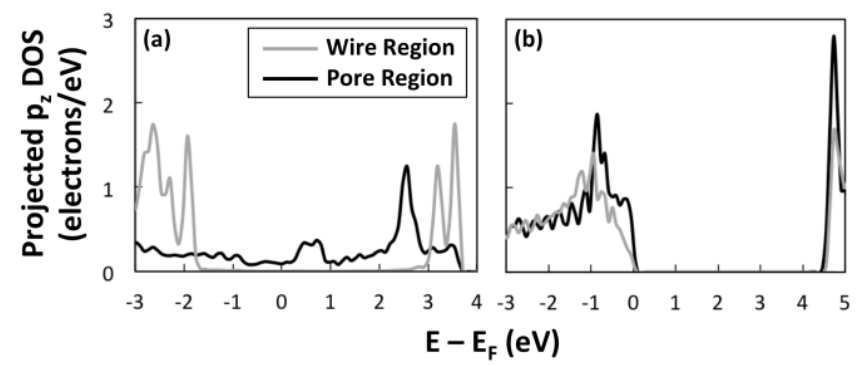

FIG. 3. Projected $p_{z}$ density of states plots based on the six highest atoms in the wire region, and the six lowest atoms in the pore region, of the $\mathrm{h}-\mathrm{BN} / \mathrm{Rh}(111)$ nanomesh in the (a) presence, and (b) absence, of the $\mathrm{Rh}(111)$ substrate.

The unequal contributions of the $\mathrm{p}_{\mathrm{z}}$ states of the low/high-lying atoms in BN to the LDOS in the vicinity of $\mathrm{E}_{\mathrm{F}}$ are attributable to the interaction of the pore atoms with the metal substrate. While the high-lying regions of the nanomesh interact only weakly with the metal via vdW attractions, the low-lying pore regions form covalent bonds with the metal surface. As the $\mathrm{p}_{\mathrm{z}}$ states of the pore region atoms re-hybridize to facilitate bonding to the substrate, they are rendered chemically distinct from the wire region atoms, and the energy of their $\mathrm{p}_{\mathrm{z}}$ states are shifted relative to those of the wire region atoms. The decisive role of the substrate-nanomesh interaction in shifting the $\mathrm{p}_{\mathrm{z}}$ states of the pore atoms is demonstrated by the changes in their PDOS when the $\mathrm{Rh}(111)$ substrate is removed while retaining the corrugated nanomesh structure. In this case the atoms in the low/high-lying regions are no longer chemically distinct, and their $\mathrm{p}_{\mathrm{z}}$ states contribute essentially equally to the LDOS (Fig. 3(b)).

The above considerations also apply to the moiré structured $g / R u(0001)$ nanomesh, where the lattice mismatch between graphene and $\mathrm{Ru}(0001)$ leads to alternating areas of low-lying "valleys" and high-lying "mounds." Here optB86b and PBE predict the geometric corrugation to be 1.45 and $1.54 \AA$ [33], in line with previous DFT values, which range from 1.2-1.7 $\AA$ [25, 26, 34]. The marked dependence of the apparent nanomesh corrugation on the STM sample voltage, which ranges from ca. 0.2 to $1.0 \AA$ as the voltage varies from +2 to $-2 \mathrm{~V}$ [35], is also reproduced by the present treatment (Fig. 2(b)). As with h-BN/Rh(111), the simulated and experimental STM apparent corrugations agree over a wide range of bias voltages, but never match the calculated geometric corrugation. Also like h-BN/Rh(111), the dominant contribution of the $g / R u(0001)$ nanomesh to the LDOS in the vicinity of $E_{F}$ comes from the $p_{z}$ states of its low-lying atoms [36]. Hence the LDOS in the vicinity of $E_{F}$ of $\mathrm{g} / \mathrm{Ru}(0001)$ are also concentrated in the low-lying regions, resulting in a diminished apparent corrugation relative to 
the geometric corrugation. What is remarkable about $\mathrm{h}-\mathrm{BN} / \mathrm{Rh}(111)$ is the magnitude of the effect, which far exceeds that observed for $\mathrm{g} / \mathrm{Ru}(0001)$. This is because monolayer $\mathrm{h}-\mathrm{BN}$ has a large band gap of $\sim 4 \mathrm{eV}$ [37] and rehybridization effectively closes the gap in the pore region (Fig. 3(a)), whereas graphene is a zero band gap semiconductor. It is also worth noting that available experimental estimates of the $g / \mathrm{Ru}(0001)$ corrugation diverge by an order of magnitude, with helium atom scattering [17], surface x-ray diffraction [18], and low energy electron diffraction [19] experiments suggesting values ranging from 0.15 to $1.5 \AA$. Clearly further experimental work is needed to conclusively resolve the structures of corrugated h-BN and graphene films.
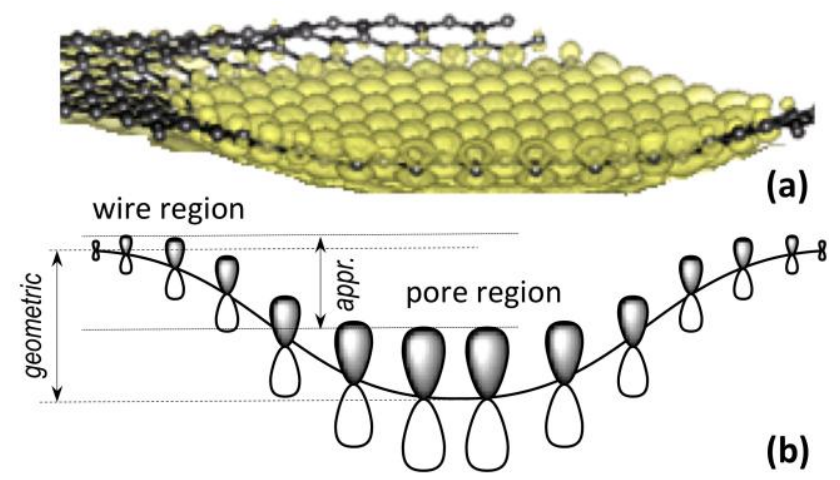

FIG. 4. (a) $0.001 \mathrm{e}^{-/ A}$ isodensity contour of the $\mathrm{h}-\mathrm{BN} / \mathrm{Rh}(111)$ nanomesh arising from the states in the $\mathrm{E}_{\mathrm{F}}$ to $\mathrm{E}_{\mathrm{F}}+1 \mathrm{eV}$ range. (b) A schematic illustrating the difference between the apparent (appr.) and the geometric corrugations.

The present findings are encapsulated in the following simple model. In effect, the $\mathrm{p}_{z}$ orbitals of the low-lying atoms in the h-BN as well as graphene nanomeshes become larger than their high-lying counterparts within a few $\mathrm{eV}$ about the $\mathrm{E}_{\mathrm{F}}$ due to re-hybridization with the metal d states. The $\mathrm{p}_{\mathrm{z}}$ orbital contours, which an STM tip follows, therefore produce an apparent corrugation that differs from the geometric corrugation (Fig. 4). As the pore region appears shallower, the apparent corrugation is reduced compared to the geometric corrugation, as is the case in the ca. $\pm 2 \mathrm{eV}$ range about $\mathrm{E}_{\mathrm{F}}$ for $\mathrm{h}-\mathrm{BN} / \mathrm{Rh}(111)$ and $\mathrm{g} / \mathrm{Ru}(0001)$, as discussed above. According to this model, the pore region can also appear higher than the wire region. This is expected to happen for $\mathrm{h}-\mathrm{BN} / \mathrm{Rh}(111)$ at a sample voltage above $\sim+2.5 \mathrm{~V}$ according to Fig. 3(a) where the LDOS of the pore region significantly outweighs that of the wire region. This "contrast inversion" is verified by the simulated STM image at $+3 \mathrm{~V}$ (Fig. 5) and has indeed been reported experimentally at $\sim+2.6 \mathrm{~V}$ and above for $\mathrm{h}-\mathrm{BN} / \mathrm{Rh}(111)$ [7]. A similar contrast inversion has been reported for $\mathrm{g} / \mathrm{Ru}(0001)[17,38]$. Furthermore, this model does not preclude the possibility of the apparent corrugation being even greater than the DFT-calculated geometric corrugation. This may be the case for e.g. $g / \operatorname{Ir}(111)[39,40]$ at certain bias voltages. 


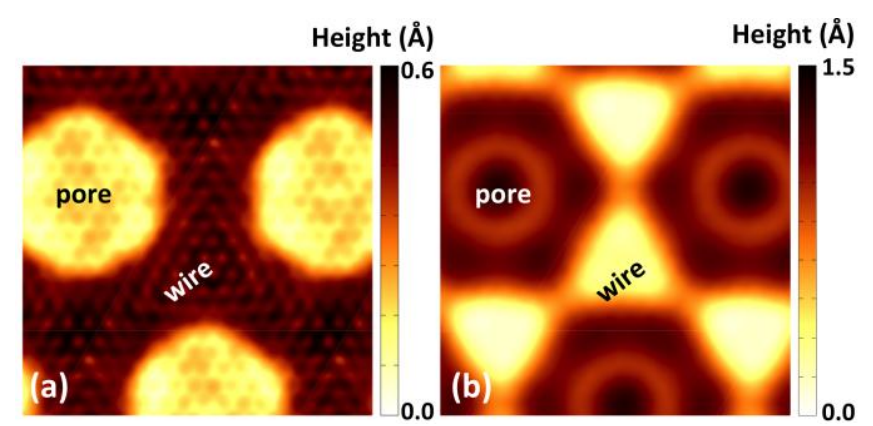

FIG. 5. Simulated STM images for h-BN/Rh(111) at a sample voltage of (a) $+1.0 \mathrm{~V}$ and (b) $+3.0 \mathrm{~V}$.

We postulate that similar effects exist for supported nanomeshes of materials besides BN and graphene. Potential candidates include $\mathrm{MoS}_{2}$ and other metal dichalcogenides, and siligraphene $\left(\mathrm{SiC}_{2}\right)$ [41, 42], although we have not been able to locate reports of experiment involving monolayers of these materials supported on metals. Other notable 2D films, such as silicene and germanene $[43,44]$, can relieve stress due to lattice mismatch by the Si and Ge atoms adopting multiple bonding arrangements, and are therefore not expected to form nanomeshes when supported on metals. In general, for nanomeshes that show signs of chemically distinct regions, e.g. splitting of bands originating from a nanomesh [4] or strong tendencies for adatoms and molecules to preferentially adsorb in specific regions of a nanomesh $[6-9,16]$, the local valence states of the chemically distinct regions of the nanomesh are likely to lie at different energies, in which case STM measurements should not be taken as a reliable indicator of the magnitude of the actual corrugation.

In conclusion, the geometric corrugations of $\mathrm{h}-\mathrm{BN} / \mathrm{Rh}(111)$ and $\mathrm{g} / \mathrm{Ru}(0001)$ as optimized using self-consistent vdW-corrected DFT calculations are fully consistent with the smaller apparent corrugations reported by STM experiments $[7,15,35]$. This is due to the localization of $\mathrm{p}_{\mathrm{z}}$ states near the Fermi level within the low regions of the nanomeshes, a phenomenon that should be heeded in the research of corrugated 2D films. Consequently, the models chosen to represent nanomesh structures in theoretical simulations, and the theoretical methods used to predict their physical and chemical properties, need to be carefully evaluated against results from experimental techniques besides STM.

\section{Acknowledgments}

Work by W.C.M. and Y. X. was performed as part of the Center for Atomic Level Catalyst Design, an Energy Frontier Research Center funded supported by the U.S. Department of Energy, Office of Science, Office of Basic 
Energy Sciences (DE-SC0001058). V.M. was supported by the Office of Naval Research. High-performance computing resources were provided by Louisiana State University (http://www.hpc.lsu.edu) and by the National Energy Research Scientific Computing Center, which is supported by the U.S. Department of Energy, Office of Science (DE-AC02-05CH11231). 


\section{References}

[1] M. Corso, W. Auwärter, M. Muntwiler, A. Tamai, T. Greber, J. Osterwalder, Science, 303 (2004) 217.

[2] S. Berner, M. Corso, R. Widmer, O. Groening, R. Laskowski, P. Blaha, K. Schwarz, A. Goriachko, H. Over, S.

Gsell, M. Schreck, H. Sachdev, T. Greber, J. Osterwalder, Angew. Chem. Int. Ed., 46 (2007) 5115.

[3] J. Wintterlin, M.L. Bocquet, Surf. Sci., 603 (2009) 1841.

[4] T. Brugger, S. Gunther, B. Wang, J.H. Dil, M.L. Bocquet, J. Osterwalder, J. Wintterlin, T. Greber, Phys. Rev. B, 79 (2009) 045407.

[5] H.G. Zhang, H. Hu, Y. Pan, J.H. Mao, M. Gao, H.M. Guo, S.X. Du, T. Greber, H.J. Gao, J. Phys.-Condens. Mat., 22 (2010).

[6] A.T. N’Diaye, S. Bleikamp, P.J. Feibelman, T. Michely, Phys. Rev. Lett., 97 (2006) 215501.

[7] I. Brihuega, C.H. Michaelis, J. Zhang, S. Bose, V. Sessi, J. Honolka, M.A. Schneider, A. Enders, K. Kern, Surf. Sci., 602 (2008) L95.

[8] A.K. Engstfeld, S. Beckord, C.D. Lorenz, R.J. Behm, ChemPhysChem, 13 (2012) 3313.

[9] M. Iannuzzi, F. Tran, R. Widmer, T. Dienel, K. Radican, Y. Ding, J. Hutter, O. Groning, Phys. Chem. Chem. Phys., 16 (2014) 12374.

[10] B. Wang, B. Yoon, M. König, Y. Fukamori, F. Esch, U. Heiz, U. Landman, Nano Lett., 12 (2012) 5907.

[11] A.K. Engstfeld, H.E. Hoster, R.J. Behm, L.D. Roelofs, X. Liu, C.-Z. Wang, Y. Han, J.W. Evans, Phys. Rev. B, 86 (2012) 085442.

[12] Y. Han, A.K. Engstfeld, R.J. Behm, J.W. Evans, J. Chem. Phys., 138 (2013) 134703.

[13] L. Semidey-Flecha, D. Teng, B.F. Habenicht, D.S. Sholl, Y. Xu, J. Chem. Phys., 138 (2013) 184710.

[14] B.F. Habenicht, D. Teng, L. Semidey-Flecha, D.S. Sholl, Y. Xu, Top. Catal., 57 (2014) 69.

[15] M.C. Patterson, B.F. Habenicht, R.L. Kurtz, L. Liu, Y. Xu, P.T. Sprunger, Phys. Rev. B, 89 (2014) 205423.

[16] Z. Zhou, F. Gao, D.W. Goodman, Surf. Sci., 604 (2010) L31.

[17] B. Borca, S. Barja, M. Garnica, M. Minniti, A. Politano, J.M. Rodriguez-García, J.J. Hinarejos, D. Farías, A.L. Vázquez de Parga, R. Miranda, New. J. Phys., 12 (2010) 093018.

[18] D. Martoccia, M. Bjorck, C.M. Schleputz, T. Brugger, S.A. Pauli, B.D. Patterson, T. Greber, P.R. Willmott, New. J. Phys., 12 (2010) 043028. 
[19] W. Moritz, B. Wang, M.L. Bocquet, T. Brugger, T. Greber, J. Wintterlin, S. Günther, Phys. Rev. Lett., 104 (2010) 136102.

[20] B. Wang, M.L. Bocquet, S. Guenther, J. Wintterlin, Phys. Rev. Lett., 101 (2008) 099703.

[21] W.A. Hofer, A.S. Foster, A.L. Shluger, Rev. Mod. Phys., 75 (2003) 1287.

[22] J. Klimeš, D.R. Bowler, A. Michaelides, Phys. Rev. B, 83 (2011) 195131.

[23] G. Kresse, J. Furthmüller, Phys. Rev. B, 54 (1996) 11169.

[24] G. Kresse, J. Joubert, Phys. Rev. B, 59 (1999) 1758.

[25] B. Wang, M.L. Bocquet, S. Marchini, S. Günther, J. Wintterlin, Phys. Chem. Chem. Phys., 10 (2008) 3530.

[26] D. Stradi, S. Barja, C. Díaz, M. Garnica, B. Borca, J.J. Hinarejos, D. Sánchez-Portal, M. Alcamí, A. Arnau, A.L. Vázquez de Parga, R. Miranda, F. Martín, Phys. Rev. Lett., 106 (2011) 186102.

[27] M. Iannuzzi, I. Kalichava, H.F. Ma, S.J. Leake, H.T. Zhou, G. Li, Y. Zhang, O. Bunk, H.J. Gao, J. Hutter, P.R. Willmott, T. Greber, Phys. Rev. B, 88 (2013) 125433.

[28] N.W. Ashcroft, N.D. Mermin, Solid State Physics, Saunders College, Orlando, FL, 1976.

[29] G. Makov, M.C. Payne, Phys. Rev. B, 51 (1995) 4014.

[30] R. Laskowski, P. Blaha, T. Gallauner, K. Schwarz, Phys. Rev. Lett., 98 (2007) 106802.

[31] J.G. Diaz, Y. Ding, R. Koitz, A.P. Seitsonen, M. Iannuzzi, J. Hutter, Theor. Chem. Acc., 132 (2013).

[32] J. Tersoff, D.R. Hamann, Phys. Rev. B, 31 (1985) 805.

[33] Z. Zhou, B.F. Habenicht, Q. Guo, Z. Yan, Y. Xu, L. Liu, D.W. Goodman, Surf. Sci., 611 (2013) 67.

[34] M. Iannuzzi, J. Hutter, Surf. Sci., 605 (2011) 1360.

[35] A.L. Vázquez de Parga, F. Calleja, B. Borca, M.C.G. Passeggi, J.J. Hinarejos, F. Guinea, R. Miranda, Phys. Rev. Lett., 100 (2008) 056807.

[36] B. Wang, S. Günther, J. Wintterlin, M.L. Bocquet, New. J. Phys., 12 (2010) 043041.

[37] Z. Huang, C. He, X. Qi, H. Yang, W. Liu, X. Wei, X. Peng, J. Zhong, J. Phys. D Appl. Phys., 47 (2014) 075301.

[38] D. Stradi, S. Barja, C. Díaz, M. Garnica, B. Borca, J.J. Hinarejos, D. Sánchez-Portal, M. Alcamí, A. Arnau, A.L. Vázquez de Parga, R. Miranda, F. Martín, Phys. Rev. B, 85 (2012) 121404.

[39] E.N. Voloshina, E. Fertitta, A. Garhofer, F. Mittendorfer, M. Fonin, A. Thissen, Y.S. Dedkov, Sci. Rep., 3 (2013) 1072. 
[40] C. Busse, P. Lazić, R. Djemour, J. Coraux, T. Gerber, N. Atodiresei, V. Caciuc, R. Brako, A.T. N’Diaye, S. Blügel, J. Zegenhagen, T. Michely, Phys. Rev. Lett., 107 (2011) 036101.

[41] Y.L. Huang, Y. Chen, W. Zhang, S.Y. Quek, C.-H. Chen, L.-J. Li, W.-T. Hsu, W.-H. Chang, Y.J. Zheng, W. Chen, A.T.S. Wee, Nat. Commun., 6 (2015) 6298.

[42] L.-J. Zhou, Y.-F. Zhang, L.-M. Wu, Nano Lett., 13 (2013) 5431.

[43] M.E. Davila, L. Xian, S. Cahangirov, A. Rubio, G. Le Lay, New. J. Phys., 16 (2014) 095002.

[44] P. Vogt, P. De Padova, C. Quaresima, J. Avila, E. Frantzeskakis, M.C. Asensio, A. Resta, B. Ealet, G. Le Lay, Phys. Rev. Lett., 108 (2012) 155501. 